\title{
Immunological aspects of infectious diseases
}

Edited by G. Dick. 1979. Lancaster, England: MTP Press Ltd. Pp. xii and 524. $£ 19.95$.

In the early days of immunology, microbiologists regarded immunologists as their natural allies and indeed it would have been hard to make a firm distinction between the two disciplines. Unhappily, this is less true of the two decades after the second world war and until recently much academic immunology has had little direct relevance to clinicians or pathologists concerned with the problems of human disease. Happily, the gap is now narrowing as immunologists return with renewed enthusiasm to studying the host response to microbial infections. This period in the wilderness was arguably salutory because immunologists have returned to problems of infection armed with a detailed knowledge of the structure and to some extent the function of the diverse components of the host immune response, which combine to limit the spread and persistance of infections. Despite the spate of books dealing with other immunological topics and their relation to disease, there have been surprisingly few dealing with the immunology of infectious diseases. Thus, both the scientific and the literary scene were set for the appearance of a book of this kind. The success of the venture is well illustrated by the fact that the majority of the topics discussed in the book do not appear in any other textbook of medical immunology. Nor are these topics contrived, because these deal directly with many immunological themes that have not been explored in the context of infectious diseases of man. These novel chapters should be mentioned first. Professor Pepys considers the whole range of allergic reactions to microbial infections and thereby provides important insight into many problems that have been long and fruitlessly debated. In particular, this chapter shows that detailed analysis of immune responses in infectious diseases can resolve the extent to which hypersensitivity contributes to tissue damage. This chapter also makes clear that the whole range of infectious agents do possess antigens that provoke allergic responses, and that additional speculation about the extent to which such allergy accounts for inflammatory disease in, for example, the respiratory and gastro-intestinal tracts, needs to be revaluated by modern techniques. Dr Bullock's discussion of anergy in infectious diseases also emphasises the important point that the extent of microbial growth in infected tissues can overwhelm the immune responses of the host by mechanisms analogous to high-dose tolerance. This concept is not only important to our understanding of chronic infectious diseases such as tuberculosis and leprosy, but is also central to any consideration of how combined chemotherapy and immunotherapy may be combined in future protocols of treatment. This theme is reinforced and elaborated in Professor Turk's analysis of immunology in a variety of chronic infections.

The other contributions to this volume are less novel but nonetheless combine to provide a body of information that has not hitherto been readily accessible to the microbiologist. The immunology of fungal infections, protozoal infections, and persistent virus infections are all considered. An important consideration in analysing immunopathological consequences of persistent infection is the realisation that the host's immune response may account for some and occasionally all the tissue damage resulting from infection. This again has important implications in terms of devising therapeutic regimes and indeed we already accept the idea that antimicrobial chemotherapy and immunosuppression may be used synergistically in some clinical situations, a concept that would have provoked horror a decade ago. In this context there are chapters dealing with immune-complex disease, autoimmunity, and slow virus infections. Finally the extent to which deficiencies in the host response can aid and abet microbial persistence is also not neglected. Immunodeficiency, whether resulting from natural causes or as the consequence of social evils, is fully reviewed.

This volume therefore contains full and well balanced contributions covering the immune response to the entire range of microbial infection of man, ways in which infectious agents can evade these mechanisms, and the extent to which the immune response itself may prove harmful to the infected host. The editor has orchestrated this performance with commendable firmness and skill and, indeed if the musical metaphor is to be pursued, it is remarkable that so many well known soloists have been persuaded to knuckle down under his authoritative baton. Clinicians and clinical pathologists will find this volume readable and often invigorating. The price is moderate and there are full lists of references for further reading.

A. M. Denman 